\title{
PERFIL SOCIODEMOGRÁFICO ASSSOCIADO EM NÍVEL DE APTIDÃO FÍSICA RELACIONADA À SAÚDE EM ESCOLARES
}

\section{Ana Paula Sehn}

Graduada em Educação Física pela Universidade de Santa Cruz do Sul (UNISC), Brasil.

\section{Cézane Priscila Reuter}

Docente do Departamento de Educação Física e Saúde da Universidade de Santa Cruz do Sul (UNISC), Brasil.

\section{Deise Graziela Kern}

Graduada em Educação Física pela Universidade de Santa Cruz do Sul (UNISC), Brasil.

\section{Cristiane Fernanda da Silva}

Graduada em Educação Física pela Universidade de Santa Cruz do Sul (UNISC), Brasil.

\section{Cláudia Daniela Barbian}

Mestranda no Programa de Pós-graduação em Promoção da Saúde (PPGPS) da Universidade de Santa Cruz do Sul (UNISC), Brasil.

\section{Letícia Welser}

Graduada em Educação Física, pela Universidade de Santa Cruz do Sul (UNISC), Brasil.

\section{Miria Suzana Burgos}

Docente do Departamento de Educação Física e Saúde e no Programa de Pós-graduação em Promoção da Saúde (PPGPS) da Universidade de Santa Cruz do Sul (UNISC), Brasil.

\section{E-mail: mburgos@unisc.br}

RESUMO: Este estudo teve como objetivo verificar se existe associação entre fatores sociodemográficos e níveis de aptidão física relacionada à saúde em escolares. Foram sujeitos deste estudo transversal 1.000 crianças e adolescentes, de sete a 17 anos de idade, de Santa Cruz do Sul-RS. Analisou-se o nível socioeconômico, o perfil nutricional, a flexibilidade, a resistência abdominal e a aptidão cardiorrespiratória (APCR). Todas as análises foram realizadas no programa estatístico SPSS v. 23.0, considerando um nível de significância de $p<0,05$. Resultados demonstraram que a prevalência de sobrepeso/obesidade foi superior no sexo masculino (RP: 1,$05 ; p=0,025)$ e inferior entre os adolescentes (RP: 0,$89 ; p<0,001$ ). Por outro lado, escolares do sexo masculino apresentam prevalência $6 \%$ menor de baixos níveis de APCR $(p=0,001)$. Adolescentes (RP: 1,$07 ; p=0,005)$, estudantes da rede municipal (RP: 1,$10 ; p=0,049)$ e da zona urbana (RP: 1,$09 ; p=0,002)$ apresentam maior prevalência de baixos níveis de APCR. Concluiu-se que fatores sociodemográficos estão associados aos baixos níveis de aptidão física em escolares.

PALAVRAS-CHAVE: Adolescente; Aptidão física; Criança.

\section{SOCIAL AND DEMOGRAPHIC PROFILE ASSOCIATED TO PHYSICAL CAPACIT Y LEVEL RELATED TO SCHOOL CHILDREN'S HEALTH}

ABSTRACT: The association between socio-demographic and physical capacity levels related to school children's health is investigated. Current transversal study comprised 1000 children and adolescents, aged between 7 and 17 years, in Santa Cruz do Sul RS Brazil. The socio-economic level, nutritional profile, flexibility, abdominal resistance and cardiorespiratory fitness (CRF) were analyzed with statistical program SPSS v. 23.0, at significance level $p<0.05$. Results demonstrated that prevalence of overweight/obesity was higher in males (PR: $1.05 ; p=0.025$ ) and lower among adolescents (PR: 0.89; $p<0.001)$. Moreover, male school children had a $6 \%$ prevalence lower than APCR low levels $(p=0.001)$. Adolescents (PR: $1.07 ; p=0.005)$, school children in public schools (PR: $1.10 ; p=0.049)$ and students from the rural area (PR: $1.09 ; p=0.002$ ) had a higher prevalence in APCR low levels. Socio-demographic factors are actually associated to low levels of physical capacity in school children.

KEY WORDS: Adolescents; Physical fitness; Child. 


\section{INTRODUÇÃO}

Atualmente, crianças e adolescentes demonstram baixos níveis de aptidão física relacionada à saúde, o que acaba refletindo na vida adulta, pois os hábitos saudáveis iniciam na infância (BARBOSA FILHO; CAMPOS; ERKELENZ et al., 2014). Estima-se que baixos níveis de aptidão física estão relacionados a alguns fatores sociodemográficos, como sexo, idade, nível socioeconômico, zona de moradia (rural e urbana), deslocamento para a escola e rede escolar (pública e privada) (BIBILONI et al., 2012; REZENDE et al., 2014; ALBERTO; FIGUEIRA JUNIOR, 2015).

Evidências apontam que o nível socioeconômico e a zona de moradia estão associados diretamente aos níveis de aptidão física, mas variam de acordo com o país (PETROSKI et al., 2012). No Brasil, estudos sugerem que indivíduosque residem nazonarural apresentam melhores níveis de flexibilidade e aptidão cardiorrespiratória; já, adolescentes com níveis socioeconômicos mais elevados tendem a demonstrar maiores níveis de sedentarismo e apresentar baixos níveis de aptidão física (COOMBS et al., 2013; DIAS et al., 2014). Na Califórnia-EUA, um estudo demonstrou que crianças e adolescentes com baixo nível socioeconômico apresentaram piores níveis de aptidão física (JIN; JONE-SMITH, 2015).

No período infantojuvenil, baixos níveis de aptidão física podem estar associados a aspectos biológico, socioeconômicos e ambientais, de modo que se torna importante e fundamental a concepção de pesquisas que evidenciem essa relação (LUGUETTI; RÉ; BÖHME, 2010), à medida que a aptidão física relacionada à saúde se caracteriza como um importante marcador de saúde em crianças e adolescentes (DAMIANI et al., 2011). Assim, este estudo tem por objetivo em verificar os níveis de aptidão física e poder contribuir para a identificação dos fatores associados às condições de saúde desta população, auxiliando na prevenção de futuros agravos e doenças cardiometabólicas que podem iniciar na adolescência e persistir na vida adulta (GONÇALVES; SILVA, 2016; TAMBALIS et al., 2013). A partir do contexto acima exposto, o presente estudo tem como objetivo verificar se existe associação entre fatores sociodemográficos e os níveis de aptidão física relacionada à saúde em escolares.

\section{MÉTODO}

Os sujeitos avaliados são 1.000 crianças e adolescentes, sendo 539 do sexo feminino, com idades entre sete e 17 anos, pertencentes a 19 escolas do município de Santa Cruz do Sul-RS. O presente estudo é recorte de uma pesquisa mais ampla, realizada no período de 2014-2015, designada "Saúde dos escolares Fase III", que foi desenvolvida pela Universidade de Santa Cruz do Sul (UNISC), aprovada pelo Comitê de Ética em Pesquisa, com Seres Humanos (CEP), sob número do parecer 714.216 .

Foi utilizado um questionário com questões referentes ao estilo de vida, saúde e bem-estar, adaptado de Barros e Nahas (2003), utilizado na pesquisa de Burgos et al. (2014). O nível socioeconômico foi avaliado pelo critério da Associação Brasileira de Empresas de Pesquisas (ABEP) (ABEP, 2012).

O perfil nutricional dos escolares foi avaliado pelo índice de massa corporal (IMC), avaliando-se o peso e a estatura, por meio da fórmula: IMC $=$ peso/altura ${ }^{2}$ $\left(\mathrm{kg} / \mathrm{m}^{2}\right)$. Na classificação, foram utilizados os pontos de corte da Organização Mundial da Saúde (WHO, 2007).

A flexibilidade do escolar foi avaliada pelo teste sentar e alcançar, utilizando o banco de Wells. O indivíduo deveria estar descalço, com os calcanhares apoiados no banco. Com os joelhos estendidos e as mãos sobrepostas, $o$ avaliado deveria se inclinar lentamente e estender as mãos para frente, mais distante possível, permanecendo, nesta posição, até ser anotado o resultado, em duas tentativas. $O$ resultado foi anotado $\mathrm{em} \mathrm{cm}$, sendo utilizado para avaliação, o melhor resultado.

A resistência muscular localizada foi avaliada pelo número de abdominais realizados em 1 minuto. $\mathrm{O}$ avaliado se posicionou em decúbito dorsal com joelhos flexionados a 45 graus e os braços cruzados sobre o peito. $\mathrm{O}$ avaliador segurou os tornozelos do aluno com as mãos, fixando os mesmos ao solo. $\mathrm{O}$ aluno executou o maior número de flexões de tronco, durante 1 minuto, sendo que para ser válido um abdominal, os cotovelos devem encostar-se às coxas; após encostar, o aluno retornou para a posição inicial e executou outra flexão.

A aptidão cardiorrespiratória foi avaliada por meio do teste corrida/caminhada de 9 minutos, sendo 
realizado em uma pista demarcada. O indivíduo foi previamente recomendado a utilizar roupas leves e calçado adequado. O resultado é obtido em metros, pela distância percorrida.

Os testes de flexibilidade, resistência muscular localizada e aptidão cardiorrespiratória são preconizados pelo PROESP-BR e os resultados de todos os testes foram categorizados da seguinte forma: 1) normal (níveis desejados) e 2) indicador de risco (níveis baixos), conforme idade e sexo (PROESP, 2009).

Foi utilizada a estatística descritiva, por meio da frequência e percentual, para caracterizar os sujeitos, sendo aplicada a regressão de Poisson, para obtenção dos valores de razão de prevalência (RP) e intervalos de confiança (IC), na comparação das variáveis preditoras (características sociodemográficas), com os desfechos (aptidão física relacionada à saúde). Todas as análises foram realizadas no programa estatístico SPSS v. 23.0 (IBM, Armonk, NY, EUA), considerando um nível de significância de $p<0,05$.

\section{RESULTADOS}

Na Tabela 1 são apresentadas as características sociodemográficas e indicadores de saúde dos escolares avaliados. Observa-se que é elevada a prevalência de escolares com baixos níveis de flexibilidade (43,3\%), resistência abdominal (48,3\%) e APCR (52,0\%), bem como com sobrepeso/obesidade (36,7\%).

Tabela 1. Caracterização dos escolares avaliados. Santa Cruz do Sul, 2011-2012

(continua)

\begin{tabular}{ll}
\hline & $\mathbf{n}(\%)$ \\
\hline Sexo & \\
Feminino & $539(53,9)$ \\
Masculino & $461(46,1)$ \\
\hline Faixa etária & \\
\hline Criança & $318(31,8)$ \\
Adolescente & $682(68,2)$ \\
\hline Rede escolar & \\
\hline Particular & $77(7,7)$
\end{tabular}

(conclusão)

\begin{tabular}{lc}
\hline & $\mathbf{n}(\%)$ \\
\hline Municipal & $336(33,6)$ \\
Estadual & $587(58,7)$ \\
\hline Zona de moradia & \\
\hline Rural & $242(24,2)$ \\
Urbana & $758(75,8)$ \\
\hline
\end{tabular}

Nível socioeconômico

\begin{tabular}{lc} 
A-B & $561(56,1)$ \\
C & $416(41,6)$ \\
D-E & $23(2,3)$ \\
\hline
\end{tabular}

Flexibilidade

\begin{tabular}{ll} 
Bons níveis & $567(56,7)$ \\
Baixos níveis & $433(43,3)$ \\
\hline
\end{tabular}

Resistência abdominal

\begin{tabular}{ll}
\hline Bons níveis & $517(51,7)$ \\
Baixos níveis & $483(48,3)$ \\
\hline IMC & $633(63,3)$ \\
\hline Baixo peso/normal & $367(36,7)$ \\
Sobrepeso/obesidade & \\
\hline Aptidão cardiorrespiratória & $480(48,0)$ \\
\hline Bons níveis & $520(52,0)$ \\
Baixos níveis &
\end{tabular}

Fonte: Dados da pesquisa

Baixos níveis de flexibilidade são mais prevalentes entre os meninos (RP: 1,16; $p<0,001$ ) e escolares da zona urbana (RP: 1,$06 ; p=0,045$ ). Para a resistência abdominal, a prevalência de baixos níveis associou-se também com o sexo masculino (RP: 1,$12 ; p<0,001$ ), bem como com a rede municipal (RP: 1,$13 ; p=0,011$ ) e estadual (RP: 1,19 ; $p<0,001$ ) de ensino (Tabela 2). 
Tabela 2. Associação entre baixos níveis de flexibilidade e resistência abdominal com características sociodemográficas

\begin{tabular}{|c|c|c|c|c|}
\hline \multirow{2}{*}{ Características sociodemográficas } & \multicolumn{2}{|c|}{ Flexibilidade } & \multicolumn{2}{|c|}{ Resistência abdominal } \\
\hline & RP (IC 95\%) & $P$ & RP (IC 95\%) & $P$ \\
\hline \multicolumn{5}{|l|}{ Sexo } \\
\hline Feminino & 1 & & 1 & \\
\hline Masculino & $1,16(1,11-1,21)$ & $<0,001$ & $1,12(1,07-1,16)$ & $<0,001$ \\
\hline \multicolumn{5}{|l|}{ Faixa etária } \\
\hline Criança & 1 & & 1 & \\
\hline Adolescente & $1,05(1,00-1,09)$ & 0,053 & $1,00(0,96-1,05)$ & 0,951 \\
\hline \multicolumn{5}{|l|}{ Rede escolar } \\
\hline Particular & 1 & & 1 & \\
\hline Municipal & $0,98(0,89-1,07)$ & 0,601 & $1,13(1,03-1,23)$ & 0,011 \\
\hline Estadual & $0,99(0,91-1,07)$ & 0,728 & $1,19(1,09-1,29)$ & $<0,001$ \\
\hline \multicolumn{5}{|l|}{ Zona de moradia } \\
\hline Rural & 1 & & 1 & \\
\hline Urbana & $1,06(1,00-1,12)$ & 0,045 & $1,00(0,95-1,06)$ & 0,882 \\
\hline \multicolumn{5}{|l|}{ Nível socioeconômico } \\
\hline $\mathrm{A}-\mathrm{B}$ & 1 & & 1 & \\
\hline $\mathrm{C}$ & $1,04(0,99-1,08)$ & 0,119 & $1,04(1,00-1,09)$ & 0,084 \\
\hline D-E & $1,04(0,90-1,20)$ & 0,581 & $0,96(0,84-1,10)$ & 0,531 \\
\hline
\end{tabular}

Regressão de Poisson considerando duas categorias para flexibilidade e resistência abdominal (baixos níveis versus bons níveis); valores significantes para $p<0,05$.

O presente estudo identificou que a prevalência de sobrepeso/obesidade é superior no sexo masculino (RP: $1,05 ; p=0,025)$ e inferior entre os adolescentes (RP: 0,89; $p<0,001)$. Por outro lado, escolares do sexo masculino apresentam prevalência $6 \%$ menor de baixos níveis de APCR $(p=0,001)$. Adolescentes (RP: 1,$07 ; p=0,005)$, estudantes da rede municipal (RP: 1,$10 ; p=0,049$ ) e da zona urbana (RP: 1,$09 ; p=0,002)$ apresentam maior prevalência de baixos níveis de APCR (Tabela 3). 
Tabela 3. Associação entre sobrepeso/obesidade e baixos níveis de aptidão cardiorrespiratória com características sociodemográficas

\begin{tabular}{|c|c|c|c|c|}
\hline \multirow[t]{2}{*}{ Características sociodemográficas } & \multicolumn{2}{|c|}{ IMC } & \multicolumn{2}{|c|}{ Aptidão cardiorrespiratória } \\
\hline & RP (IC 95\%) & $P$ & RP (IC 95\%) & $P$ \\
\hline \multicolumn{5}{|l|}{ Sexo } \\
\hline Feminino & 1 & & 1 & \\
\hline Masculino & $1,05(1,01-1,10)$ & 0,025 & $0,94(0,90-0,97)$ & 0,001 \\
\hline \multicolumn{5}{|l|}{ Faixa etária } \\
\hline Criança & 1 & & 1 & \\
\hline Adolescente & $0,89(0,85-0,93)$ & $<0,001$ & $1,07(1,02-1,11)$ & 0,005 \\
\hline \multicolumn{5}{|l|}{ Rede escolar } \\
\hline Particular & 1 & & 1 & \\
\hline Municipal & $0,99(0,90-1,09)$ & 0,849 & $1,10(1,00-1,20)$ & 0,049 \\
\hline Estadual & $0,99(0,91-1,08)$ & 0,799 & $1,08(0,99-1,17)$ & 0,085 \\
\hline \multicolumn{5}{|l|}{ Zona de moradia } \\
\hline Rural & 1 & & 1 & \\
\hline Urbana & $1,02(0,97-1,08)$ & 0,444 & $1,09(1,03-1,14)$ & 0,002 \\
\hline \multicolumn{5}{|l|}{ Nível socioeconômico } \\
\hline$A-B$ & 1 & & 1 & \\
\hline $\mathrm{C}$ & $0,96(0,92-1,01)$ & 0,119 & $0,97(0,93-1,01)$ & 0,189 \\
\hline D-E & $1,07(0,93-1,23)$ & 0,329 & $0,98(0,85-1,13)$ & 0,792 \\
\hline
\end{tabular}

IMC: índice de massa corporal; regressão de Poisson considerando duas categorias para IMC (baixo peso/normal versus sobrepeso/obesidade) e aptidão cardiorrespiratória (baixos níveis versus bons níveis); valores significantes para $p<0,05$.

\section{DISCUSSÃO}

No presente estudo, o sexo masculino (RP: $1,16 ; p<0,001)$ e escolares de zona urbana (RP: 1,06 ; $p=0,045$ ) apresentaram maior prevalência de baixos níveis de flexibilidade. Diferentemente, em um estudo realizado com adolescentes na Eslováquia, observouse que indivíduos do sexo masculino, da zona urbana alcançam melhores níveis de desempenho nos testes de flexibilidade, em comparação a moradores da zona rural (BEBCAKOVA et al., 2015). Na Europa, um estudo com crianças demonstrou que no sexo feminino houve melhores níveis de flexibilidade, em comparação ao sexo masculino (ETAYO et al., 2014).

Outro estudo realizado com adolescentes de escolas estaduais em Minas Gerais apontou que indivíduos da zona urbana têm 56\% mais chances de apresentarem baixos níveis de flexibilidade do que escolares da zona rural (PETROSKI et al., 2012). Por outro lado, na Colômbia, os adolescentes do sexo masculino apresentaram melhores índices de flexibilidade do que o sexo feminino (BENAVIDES; BAUTISTA; VÉLEZ, 2015). Em um estudo realizado por Dumith, Azevedo Júnior e Rombaldi (2008), com adolescentes de ambos os sexos, da cidade de Rio Grande (RS), no sexo feminino obteve melhores índices de flexibilidade que no sexo masculino, não diferindo na comparação entre escola pública e privada.

Para a resistência abdominal, os dados do presente estudo demonstram que baixos níveis são mais prevalentes, no sexo masculino (RP: 1,$12 ; p<0,001)$ e na rede municipal (RP: 1,$13 ; p=0,011)$ e estadual (RP: 1,19 ; $p<0,001)$ de ensino. Para a zona de moradia, não foram observadas diferenças significativas. Diferentemente, na cidade de Rio Grande- RS, um estudo realizado com adolescentes demonstrou que os indivíduos residentes 
da zona urbana tiveram melhores níveis de resistência abdominal que os residentes da zona rural (DUMITH; AZEVEDO JÚNIOR; ROMBALDI, 2008). Na Eslováquia, um estudo realizado com adolescentes observou que indivíduos do sexo masculino, residentes de zona urbana, alcançou melhores níveis de desempenho, no teste de força e resistência abdominal, quando comparados a moradores de zona rural (ANDRADE et al., 2014). Em Minas Gerais, um estudo realizado com adolescentes de escolas estaduais demonstrou que escolares da zona rural têm dez vezes mais chances de apresentarem baixos níveis de resistência abdominal, do que indivíduos da zona urbana (PETROSKI et al., 2012).

No presente estudo, no sexo masculino ocorreu maior prevalência de sobrepeso/obesidade (RP: 1,05; $\mathrm{p}=0,025)$, mas os adolescentes demonstraram menor prevalência desta condição, do que as crianças (RP: 0,89; $\mathrm{p}<0,001)$. Já, em um estudo realizado na cidade de Rio Grande (RS), com adolescentes de ambos os sexos, demonstrou que o IMC não apresentou diferenças para os sexos e nem entre as escolas públicas e privadas (DUMITH; AZEVEDO JÚNIOR; ROMBALDI, 2008). Não foram observadas diferenças significativas, na comparação entre as zonas de moradia. Por outro lado, no Equador, um estudo realizado com adolescentes identificou que moradores de zona urbana apresentam IMC mais elevado que residentes de zona rural (ANDRADE et al., 2014). Em outra pesquisa, realizada com crianças e adolescentes de ambos os sexos, na Argentina, pode-se observar que crianças do sexo feminino tinham maior prevalência de sobrepeso que o sexo masculino, diferentemente dos adolescentes, que apresentaram maior risco de sobrepeso, para o sexo masculino (SECCHIA et al., 2014).

Já, no teste de APCR, no sexo feminino ocorreram baixos níveis (prevalência 6\% maior; $p<0,001$ ); como também os adolescentes (RP: 1,$07 ; p=0,005$ ), os estudantes da rede municipal (RP: 1,$10 ; p=0,049$ ) e da zona urbana (RP: 1,$09 ; p=0,002$ ). Na Argentina, uma pesquisa realizada com crianças e adolescentes demonstrou que, no sexo masculino, houve melhores índices de aptidão cardiorrespiratória, que no sexo feminino e $31,6 \%$ dos indivíduos demonstraram ter baixos níveis de aptidão cardiorrespiratória (SECCHIA et al., 2014). Outro estudo realizado com adolescentes de ambos os sexos, da cidade de Rio Grande (RS), apontou que os meninos apresentaram melhores níveis de aptidão cardiorrespiratória do que as meninas, não observando diferença entre as escolas (DUMITH; AZEVEDO JÚNIOR; ROMBALDI, 2008).

Em Minas Gerais, um estudo com adolescentes demonstrou que os indivíduos da zona urbana possuem 91\% mais chances de apresentarem baixos níveis de aptidão cardiorrespiratória, em comparação a indivíduos da zona rural (PETROSKI et al., 2012). Um estudo realizado na Europa, com crianças, encontrou melhores níveis de aptidão cardiorrespiratória, para o sexo masculino, em comparação com o sexo feminino (ETAYO et al., 2014). Outro estudo realizado com adolescentes de ambos os sexos em Londrina-PR demonstrou que existe associação entre aptidão cardiorrespiratória e o sexo feminino para idade inferior a 14 anos; diferindo para idade superior a 14 anos, que a associação foi com o sexo masculino (COLEDAM et al., 2016).

Em Criciúma (SC), uma pesquisa, realizada com adolescentes de ambos os sexos, identificou baixos níveis de APCR, no sexo masculino, em escolares de rede pública de ensino e com nível econômico médio (OLIVEIRA et al., 2012). Os resultados encontrados em um estudo realizado em Jacarezinho- PR, com crianças e adolescentes de dez a 18 anos, demonstraram que, para ambos os sexos, quanto maior a faixa etária, menos ativos eram os adolescentes (SANTOS et al., 2014).

\section{CONCLUSÃO}

Conclui-se que fatores sociodemográficos estão associados aos baixos níveis de aptidão física relacionada à saúde em escolares. Observou-se que a flexibilidade esteve associada com os escolares de zona urbana e com o sexo masculino; a resistência abdominal com meninos e escolares de rede municipal e estadual de ensino e a APCR associou-se ao sexo feminino, rede municipal de ensino e zona urbana. A prevalência de sobrepeso/ obesidade foi superior no sexo masculino e nas crianças. 


\section{REFERÊNCIAS}

ABEP. Associação Brasileira de Empresas de Pesquisa. Critério de classificação econômica Brasil. 2012. Disponível em: <http://www.abep.org/criterio-brasil > Acesso em: 18 mar. 2016.

ALBERTO, A. A. D.; FIGUEIRA JUNIOR, A. J. Prevalência de inatividade física e sua associação com variáveis sociodemográficas em adolescentes do Município de Macapá/AP. Rev Bras Ciênc Mov., v. 23, n. 4, p. 80-93, 2015.

ANDRADE, S.; OCHOA-AVILÉS, A.; LACHAT, C.; ESCOBAR, P.; VERSTRAETEN, R.; VAN CAMP, J; DONOSO, S.; ROJAS, R.; CARDON, G.; KOLSTEREN, P. Physical fitness among urban and rural Ecuadorian adolescents and its association with blood lipids: a cross sectional study. BMC Pediatrics, v. 14p. 1-10, 2014.

BARBOSA FILHO, V. C.; CAMPOS W.; LOPES, A. S. Epidemiology of physical inactivity, sedentary behaviors, and unhealthy eating habits among Brazilian adolescents: a systematic review. Ciênc Saúde Colet., v.19, n.1, p.173193, 2014.

BARROS, M. V. G.; NAHAS, M. V. Medidas da atividade física: teoria e aplicação em diversos grupos populacionais. Londrina: Miodiograf, 2003.

BEBCAKOVA, V.; VADASOVA, B.; KACUR, P.; JUNGER, J.; BORZIKOVA, I.; ZVONAR, M; GIMUNOVA, M. Distribution of health-related physical fitness in Slovak population. Springer Plus, v. 4, p. 1-8, 2015.

BENAVIDES, D. H. P.; BAUTISTA, J. E. C.; VÉLEZ, R. Niveles de actividad física, condición física y tiempo en pantallas em escolares de Bogotá, Colombia: estudio FUPRECOL. Nutr Hosp, v. 32, n. 5, p. 2184-2192, 2015.

BIBILONI, M. M.; PICH, J.; CÓRDOVA, A.; PONS, A.; TUR, J. A. Association between sedentary behaviour and socioeconomic factors, diet and lifestyle among the Balearic Islands adolescents. BMC Public Health, v. 12, p. 1-1, 2012.

BURGOS, M. S. Saúde dos Escolares - Fase III: Avaliação de indicadores bioquímicos, genéticos, hematológicos, imunológicos, posturais, somotomotores, saúde bucal, fatores de risco às doenças cardiovasculares e estilo de vida de escolares: estudo em Santa Cruz do Sul-RS (Projeto de Pesquisa). Santa Cruz do Sul: UNISC, 2014.

COLEDAM, D. H. C.; FERRAIOL, P. F.; SANTOS, J. W.; OLIVEIRA, A. R. Fatores associados à aptidão cardiorrespiratória de escolares. Rev Bras Med Esporte, v. 22, n. 1, p. 21-26, 2016.

COOMBS, N.; SHELTON, N., ROWLANDS, A.; STAMATAKIS, E. Children's and adolescents' sedentary behaviour in relation to socioeconomic position. $\mathbf{J}$ Epidemiol Community Health, v. 67, n. 10, p. 868-874, 2013.

DAMIANI, D. et al. Síndrome metabólica em crianças e adolescentes: dúvidas na terminologia, mas não nos riscos cardiometabólicos. Arq Bras Endocrinol Metabol., São Paulo, v. 55, n. 8, p. 576-582, 2011.

DIAS, P.J.P.; DOMINGOS, I. P.; FERREIRA, M. G.; MURARO, A. P.; SICHIERI, R.; SILVA, R. M. G. Prevalence and factors associated with sedentary behavior in adolescents. Rev Saúde Pública, v. 48, n. 2, p. 266-274, 2014.

DUMITH, S. C.; AZEVEDO JÚNIOR, M. R.; ROMBALDI, A. J. Aptidão Física Relacionada à Saúde de Alunos do Ensino Fundamental do Município de Rio Grande, RS, Brasil. Rev Bras Med Esporte, v. 14, n. 5, p. 454-459, 2008.

ERKELENZ, N.; SCHREIBER, A. C.; KOBEL, S.; KETTNER, S.; DRENOWATZ, C.; STEINACKER, J. M. Relationship of parental health-related behaviours and physical fitness in girls and boys. Z Gesundh Wiss, v. 22, n. 5, p. 407-414, 2014.

MIGUEL-ETAYO, P.; GARCIA-MARCO, L.; ORTEGA, F. B.; INTEMANN, T.; FORAITA, R.; LISSNER, L.; OJA, L.; BARBA, G.; MICHELS, N.; TORNARITIS, M.; MOLNÁR, D.; PITSILADIS, Y.; AHRENS, W.; MORENO, L. A.; IDEFICS CONSORTIUM. Physical fitness reference standards in European children: the IDEFICS study. Int J Obes (Lond)., v. 38, p. 57-66, 2014.

GONÇALVES, E. C. A.; SILVA, D. A. S. Prevalência e fatores associados a baixos níveis de aptidão aeróbia em 
adolescentes. Rev Paul Pediatr., São Paulo, v. 34, n. 2, p. 141-47, 2016.

JIN, Y.; JONES-SMITH, J. C. Associations between family income and children's physical fitness and obesity in California, 2010-2012. Prev Chronic Dis, v. 12, p. 1-9, 2015.

LUGUETTI, C. N.; RÉA. H. N.; BÖHME, M. T. S. Indicadores de aptidão física de escolares da região centro-oeste da cidade de São Paulo. Rev. bras. cineantropom. desempenho hum., Florianópolis, v. 12, n. 5, p. 331337, 2010.

OLIVEIRA, G.; SILVA, D. A. S.; MAGGI, R. M.; PETROSKI, E. L.; FARIAS, J. M. Fatores sociodemográficos e de aptidão física associados a baixos níveis de atividade física em adolescentes de uma cidade do Sul do Brasil. Rev Educ Fís UEM, v. 23, n. 4, p. 635-645, 2012.

PETROSKI, E. L.; SILVA, A. F.; RODRIGUES, A. B.; PELEGRINI, A. Associação entre baixos níveis de aptidão física e fatores sociodemográficos em adolescentes de área urbanas e rurais. Motriz, v. 8, n. 1, p. 5-13, 2012.

PROESP-BR. Projeto Esporte Brasil. Manual. 2009. Disponível em: http://www.proesp.ufrgs.br Acesso em: 18 de março de 2016.

REZENDE, L. F. M.; AZEREDO, C. M.; CANELLA, D. S.; CLARO, R. M.; CASTRO, I. R. R; LEVY, R. B.; LUIZ ODO, C. Sociodemographic and behavioral factors associated with physical activity in Brazilian adolescents. BMC Public Health, v. 14, p.1-11, 2014.

SANTOS, G. C.; STABELINI NETO, A.; SENA, J. S.; CAMPOS, W. Atividade física em adolescentes: uma comparação entre os sexos, faixas etárias e classes econômicas. Rev Bras Ativ Fís Saúde, v.19, n. 4, p. 455-464, 2014.

SECCHIA, J. D.; GARCÍA, G. C.; ESPAÑA-ROMERO, V.; CASTRO-PIÑERO, J. Condición física y riesgo cardiovascular futuro en niños y adolescentes argentinos: una introducción de la batería ALPHA. Arch Argent Pediatr., v. 112, n. 2, p.132-140, 2014.

TAMBALIS, K. D.; PANAGIOTAKOS, D. B.; ARNAOUTIS, G.; SIDOSSIS, L. S. Endurance, explosive power and muscle strength in relation to Body Mass Index and physical fitness in Greek children aged 7 to 10-y-old. Pediatr Exerc Sci, v. 25, n. 3. p. 394-406, 2013.

WHO. World Health Organization. Growth reference data for 5-19 years. 2007. Disponível em: http://www.who.int/ growthref/en/ . Acesso em: 21 mar. 2016.

Recebido em:

Aceito em: 31 de maio de 2017 